\title{
Ejecutivo y legislativo: la inacabada disputa del poder en el sistema político Ecuatoriano.
}

Executive and legislative branches: the unfinished dispute of power in the Ecuadorian political system.

Dahik Astudillo Ivonne. ${ }^{1}$, Del Pozo Durango Rodrigo. ${ }^{2}$, Galarza Juan Manuel. ${ }^{3}$ \& Rivadeneira Ramos Edgar. ${ }^{4}$

Recibido: 03-12-2017 / Revisado: 09-02-2018 Aceptado:18-03-2018/ Publicado: 01-04-2018

\begin{abstract}
.
DOI: https://doi.org/10.33262/cienciadigital.v2i2.82

Political instability and numerous governance crises marked the return of democracy in Ecuador from 1979 to the present. In fact, three presidents did not finish their term due to social pressures or parliamentary beatings (Pérez Liñán, 2009). From this situation of political instability, product of presidentialism as a regime and its conflicting relationship from the Ejecutive with the Legislative, several studies converge that outline the need for a strong and institutionalized party system, as a mechanism that allows to carry out clear policies and predictable, in addition to granting political actors the ability to be co-accountable to the electorate in a democratic scenario with electoral rules characterized by allowing open and competitive elections. In this context, the present study intends, in the first instance, to describe Ecuadorian presidentialism. Next, we will contextualize the Ecuadorian political system to, finally, analyze the impeachment of former President Bucaram in 1996 by the Legislature; as an example of the unfinished dispute over power in Ecuador
\end{abstract}

Keywords: Presidentialism, Parliamentarism, Political Systems, Ecuador.

\section{Resumen.}

${ }^{1}$ Universidad Estatal de Bolívar, Bolívar, Ecuador, jgalarza@ueb.edu.ec

2 Universidad Estatal de Bolívar, Bolívar, Ecuador, rdurango1973@yahoo.es

3 Universidad Estatal de Bolívar, Bolívar, Ecuador, jgalarza@ueb.edu.ec

${ }^{4}$ Universidad Estatal de Bolívar, Bolívar, Ecuador, eribaden@yahoo.com 
La inestabilidad política y las numerosas crisis de gobernabilidad marcaron el retorno de la democracia en el Ecuador desde 1979 hasta la fecha. En los hechos, tres presidentes no terminaron su mandato por presiones sociales o golpes parlamentarios (Pérez Liñán, 2009). A partir de esta situación de inestabilidad política -producto del presidencialismo como régimen y la conflictiva relación entre el Ejecutivo con el Órgano legislativo- convergen varios estudios que esbozan en la necesidad de un sistema de partidos fuerte e institucionalizado; como mecanismo que permita llevar a cabo políticas claras y previsibles, además de otorgarles a los actores políticos la capacidad de ser corresponsables (accountability) frente al electorado en un escenario democrático con reglas electorales caracterizadas por permitir elecciones abiertas y competitivas. En ese contexto, el presente estudio pretende, en primera instancia, describir el presidencialismo ecuatoriano. Seguidamente, contextualizaremos el sistema político ecuatoriano para, finalmente, analizar la destitución del ex presidente Bucaram en 1996 por parte del Legislativo; como ejemplo de la inacabada disputa por el poder en el Ecuador.

Palabras Claves: Presidencialismo, parlamentarismo, sistemas políticos, Ecuador.

\section{Introducción.}

\section{Presidencialismo en el Ecuador.}

El Ecuador es un país presidencialista desde el inicio de su historia como República, por supuesto con las debidas variaciones en su sistema político propuesto en las veinte Constituciones, hasta la fecha. Actualmente, la Constitución de Montecristi ${ }^{5}$, entre uno de los más importantes cambios y que merece anotarlo debido al objetivo de este trabajo académico en consideración que subyace a la destitución de Bucaram, es el de la posibilidad del Ejecutivo y del Legislativo de destituirse y disolverse mutuamente; facultades establecidas en los artículos 130 y 148 de la Constitución de 2008. Siguiendo la idea, podría ocurrir, que estos artículos mencionados serían una consecuencia histórica de la tensa relación y, a la disputa, entre el Ejecutivo y el Legislativo por el poder, en definitiva, una respuesta a la realidad del sistema político en el Ecuador.

Sin embargo, Nohlen (1991) señala que el éxito de un sistema político dependería, no tanto del modelo que se adopta o de la normativa implementada, sino de la medida en que ésta se adapta a las necesidades del caso. Es decir, a la importancia relativa de los factores del contexto, sean estos culturales, sociológicos u otros. De acuerdo con ello, sin embargo, la realidad ecuatoriana muestra la necesidad de leyes e instrumentación constitucional debido a lo ocurrido en el caso de la destitución de Bucaram, por ejemplo, y la manipulación del

\footnotetext{
5 Nos referimos a la Constitución de la República del Ecuador. "Dejemos el pasado atrás", publicada en el Registro Oficial № 449 del 20 de octubre de 2008.
} 
instrumento legal para lograrlo por parte del Congreso en 1997; hecho que será abordado más adelante y que es uno de los propósitos de este trabajo académico.

Es por este motivo que, en la mayoría de los textos constitucionales ecuatorianos y ratificados en la Constitución de Montecristi, se ha evidenciado una categorización del presidencialismo ecuatoriano como extremadamente fortalecido o hiper-presidencialista (Salgado, 2013). Esta característica, como uno de los factores que genera mayor conflictividad del Ejecutivo con los otros poderes del Estado, principalmente con el Legislativo, ha sido calificada como acartonada y ortopédica rigidez del presidencialismo (Sartori,1992). Según Forewaker (2003), el plazo de las funciones del Presidente es fatal ${ }^{6}$, mientras que Linz (1994), determina como causa del conflicto a esa rigidez manifiesta, sumándose Duverger (1970) cuando esboza, a esta rigidez, como el producto del incremento de los poderes del régimen presidencial clásico en detrimento de las atribuciones parlamentarias de los Órganos legislativos, siendo esta deficiencia del presidencialismo una de las causantes el colapso de las democracias de América Latina y, concretamente, en Ecuador. Al respecto Payne (2006) va más allá y expone su pesimismo sobre la viabilidad del presidencialismo argumentando que se configura un escenario en el cual: a) el desarrollo de las elecciones generales en dos diferentes momentos conlleva a un retraso político, además que, b) en los regímenes presidencialistas, en donde los períodos son fijos, los legisladores suelen crear inestabilidad y es esta paralización política la que provoca la rigidez del sistema. En ese contexto, el Presidente (Ejecutivo en adelante) al ser elegido en un ámbito nacional, influye y adquiere mayor legitimidad sobre los votantes, evitándose de esta manera la tarea de negociar con la oposición o permitirse retroceder ante su propuesta de agenda de políticas públicas e intereses, ya que sus decisiones son tomadas según Linz (1993) por los poderes institucionales otorgados; la estructuración del gabinete de Ministros y la Administración Pública con representación en los territorios no necesita la aprobación parlamentaria.

Esta configuración tiende a convertir, al ejercicio democrático, en un juego de todos pierden o de suma cero (Linz, 1993); ya que separa al Ejecutivo del anclaje del Legislativo y otros poderes políticos, asumiendo la titularidad de cada uno de estos poderes para constituirse -no solamente- en un gobierno de poderes separados (Valles,op.cit) (Linz, op.cit) sino en un régimen compuesto por instituciones desarticuladas que comparten el poder (Valles,op.cit.) e incluso, compitiendo entre ellas por él (Sánchez, 2008). En este sentido, se configura una legitimidad doble (Linz,1993), cuando los dos poderes reclaman su legitimidad a través del voto popular, o en definitiva, al pueblo mismo (Balda, 2008). Esta circunstancia produce, eventualmente, que el Ejecutivo y la agenda de políticas

${ }^{6}$ El Presidencialismo en América Latina, según Foweraker (2003), se adopta a través de a) un sistema electoral con la regla de elecciones presidenciales en segunda vuelta (ballotage); b) la exclusiva censura a ministros; c) la posibilidad de disolver al Congreso; d) reelección presidencial variable en cada País; incluso algunos planteando la reelección indefinida; y e) el dominio de las comisiones legislativas en el Congreso. 
públicas planteada por este se aísle; debido a los gobiernos divididos y a la ausencia de un bloque mayoritario que impulse en el Legislativo su propuesta. Así, comparativamente y siguiendo a Linz (op.cit.), el parlamentarismo entraría en una suerte de ventaja frente al presidencialismo, debido a su flexibilidad y al contexto institucional que permiten formalizar y robustecer la democracia.

Igualmente, Nohlen (1991) plantea la tesis a favor del parlamentarismo, cuando le entrega a esta forma de gobierno- una mayor facultad para tomar decisiones consensuadas en contraposición al disenso, que apunta el autor al caracterizar al presidencialismo. Así, se atribuye al presidencialismo con el Ejecutivo a la cabeza, la construcción de la agenda de políticas públicas, mientras que el parlamentarismo, presupondría una colaboración entre Legislativo y Ejecutivo para hacerlo. Esto podría lograrse en un régimen presidencialista a través de a) un gobierno unificado, b) por medio de un triunfo electoral al conseguir una mayoría absoluta de legisladores del partido del Ejecutivo en el Legislativo, o a la inversa, es decir concentrando los poderes legislativos en el Ejecutivo (Colome y Negretto, 2003).

La tercera vía, que permitiría alcanzar una gobernanza -en este esquema de poderes divididos- radicaría en otorgarle un papel protagónico al Legislativo (Ibídem). Algo difícil de concebir ya que en esta pugna de poderes se ha evidenciado como actor principal al Legislativo, quien muchas veces ha: a) obstruido la agenda de políticas públicas y de gobierno propuesto por el Ejecutivo; b) trabado leyes y; c) tomado la decisión final al terminar abruptamente el periodo de gobierno; como el caso de Bucaram que abordaremos más adelante, provocando una crisis presidencial con casos graves de "conflictos ente el Ejecutivo y el Legislativo en los cuales una de las ramas electas de gobierno busca la disolución de la otra"(Liñán, 2009: 84).

\section{Caracterización del sistema político ecuatoriano.}

La mayor parte de estudios sobre el desempeño del sistema político ecuatoriano y, más concretamente, del sistema de partidos políticos en los veinticinco años transcurridos desde el retorno a la democracia en 1979 se han concentrado en los aspectos negativos o, en general, en sus deficiencias. Los estudios muestran una a) débil institucionalización (Mainwaring y Scully, 1995), (Bustamante, 1999), (Conaghan, 1994), b) un constante cambio de las reglas electorales (Pachano, 2003a), c) dispersión, fragmentación y atomización (Conaghan, 1995; Freidenberg, 2000), d) una creciente inestabilidad y volatilidad (Mainwaring y Scully, op.cit.), y, e) poca capacidad de representación (Mejía, 2002), como las características centrales. Así mismo, los estudios revisados han puesto

énfasis en las prácticas clientelares y corporativas a las que se han adaptado los partidos (Bustamante, 1997; Pachano, 2003a).

En ese contexto, en primer lugar, el constante cambio de las normas constitucionales, de la ley de elecciones, de partidos políticos y las reglas electorales, sin duda, han afectado 
negativamente a la estabilidad del sistema democrático y, consecuentemente a los partidos, con ello a la dinámica política e institucionalización (Valles et. al., 2015). Es así que, la permanencia y la regularidad de las normas que rigen a un sistema democrático, se consideran elementos básicos para su institucionalización en la medida en que establecen las pautas de conducta que, tanto electores como partidos, deben desarrollar para garantizar las condiciones de la competencia política (Mainwaring y Scully, 1995).

Siguiendo esta línea, se radicaliza la importancia de la estabilidad de las reglas como elemento fundamental para la institucionalización del sistema de partidos, junto al establecimiento de fuertes raíces de los partidos en la sociedad, la legitimidad otorgada por los principales actores, tanto al proceso electoral como a los partidos y la organización autónoma de estos en relación a grupos de presión y a los intereses de los líderes (Ibídem). En sentido contrario a esta necesidad, el sistema de partidos ecuatoriano, conjuntamente con el sistema electoral, han sobrellevado constantes modificaciones, algunas de las cuales podrían interpretarse incluso como acciones de sustitución de un sistema por otro. Sin embargo, está claro que es el voto popular es el que produce y otorga legitimidad a los poderes del Estado en un régimen democrático; dicho modo de producción y los procedimientos por los cuales los votos se traducen en representación política (Nohlen,1993) se encuentran siempre regulados por un determinado sistema de partidos y un sistema electoral ${ }^{7}$.

Es así que desde 1983, espacio temporal anterior a que se cumpliera el primer período presidencial y legislativo desde el retorno a la democracia en 1979, se han revisado reiteradamente las leyes que rigen a las elecciones y a los partidos políticos, igualmente las que determinan la estructura y funcionamiento del poder Legislativo. Igualmente, se han introducido múltiples reformas a la Constitución de 1979, ya sea por medio de reformas legales procesadas por la vía legislativa o por plebiscito.

De esta manera, más que los ajustes y las reformas que deben realizarse a lo largo del tiempo para adecuar las condiciones institucionales a las necesidades de la actividad política, se ha procurado transformar esas condiciones y la propia conformación de las instituciones representativas (Conaghan, 1995). Como resultado, el país no ha contado con períodos relativamente largos de vigencia de un cuerpo normativo que pudiera ser puesto a prueba en procesos de mediano alcance $\mathrm{y}$, que, a la vez, ofreciera algún grado de certidumbre en tanto instrumento para las prácticas políticas, especialmente para la acción de los partidos y su relación con los electores.

En segundo lugar, el número elevado de partidos políticos que participan en las elecciones y que obtienen escaños legislativos en el espacio temporal comprendido entre 1979 y 1996

\footnotetext{
7 Véase Nohlen (1993) para visualizar la incidencia de los sistemas electorales en el sistema político y de partidos en el ordenamiento de la representación política y en el tipo de competencia o cultura política.
} 
(según puede observarse en el Gráfico $\mathrm{N}^{\mathrm{o}}$ 1), es un indicador claro de la dispersión y de la fragmentación del sistema ${ }^{8}$.

Gráfico $\mathbf{N}^{\mathbf{0}}$ 1. Partidos políticos que participan en las elecciones.

\begin{tabular}{cccc}
\hline $\begin{array}{c}\text { Año de } \\
\text { elecciones }\end{array}$ & $\begin{array}{c}\text { Número de } \\
\text { partidos políticos }\end{array}$ & $\begin{array}{c}\text { Número de } \\
\text { legisladores }\end{array}$ & $\begin{array}{c}\text { Promedio de } \\
\text { legisladores por } \\
\text { partido }\end{array}$ \\
\hline 1979 & 10 & 69 & 6.9 \\
1984 & 15 & 71 & 4.7 \\
1986 & 14 & 71 & 5.1 \\
1988 & 12 & 71 & 5.9 \\
1990 & 11 & 72 & 6.5 \\
1992 & 13 & 77 & 5.9 \\
1994 & 14 & 77 & 5.5 \\
1996 & 11 & 82 & 7.5 \\
\hline
\end{tabular}

Fuente: Consejo Nacional Electoral del Ecuador (CNE).

El alto número de partidos políticos y sus listas con representación parlamentaria se ve reflejado en la presencia de un número significativo de bancadas pequeñas, las cuales tienen tres legisladores o menos (según puede visualizarse en el Gráfico $\mathrm{N}^{\circ} 2$ ), y que han predominado en el Legislativo desde 1979 hasta 1996; para efectos del trabajo actual hemos tomado este último año como referencia, en donde la Primera Bancada del Legislativo fue el Partido Social Cristiano (PSC en adelante) con 32,9\% siendo su tendencia política de oposición al ganador del proceso electoral, esto es Abdalá Bucaram.

Gráfico $\mathbf{N}^{\mathbf{0}} 2$ Distribución porcentual de partidos por número de escaños.

\begin{tabular}{cccccccccc}
\hline $\begin{array}{c}\text { Año/\% } \\
\text { bancadas }\end{array}$ & $\mathbf{1}$ & $\mathbf{2}$ & $\mathbf{3}$ & $\mathbf{4}$ & $\mathbf{5}$ & $\mathbf{6}$ & $\mathbf{7}$ a 10 & $\mathbf{1 0}$ a $\mathbf{2 0}$ & $\mathbf{2 1}$ o más \\
\hline 1979 & 30.0 & 50.0 & 60.0 & 70.0 & 70.0 & 70.0 & 80.0 & 90.0 & 100.0 \\
1984 & 26.7 & 40.0 & 28.6 & 50.0 & 27.3 & 46.2 & 57.2 & 45.5 & 100.0 \\
1986 & 28.6 & 28.6 & 50.0 & 64.3 & 71.4 & 78.5 & 85.6 & 99.9 & 100.0 \\
1988 & 25.0 & 50.0 & 50.0 & 58.3 & 58.3 & 66.6 & 91.6 & 91.6 & 100.0
\end{tabular}

\footnotetext{
8 Desde 1979 han participado en cada elección un mínimo de 9 listas, tanto de partidos como de candidatos independientes, con representación en el Congreso. Esto quiere decir que 12.8 partidos políticos diferentes, en promedio, han ocupado escaños legislativos, lo que constituye un número muy alto para un Congreso relativamente pequeño como es el ecuatoriano (cuyo tamaño promedio ha sido de 81.3 diputados a lo largo del periodo). Desde la perspectiva de cada período legislativo esto se muestra en que el tamaño promedio máximo ha sido de 13.7 diputados por bancada (en 1998) y el mínimo de 4.7 (en 1984), con un promedio total de 6.2.
} 


\begin{tabular}{cccccccccc}
1990 & 9.1 & 27.3 & 54.6 & 54.6 & 54.6 & 54.6 & 72.8 & 100.0 & 100.0 \\
1992 & 30.8 & 46.2 & 53.9 & 53.9 & 61.6 & 69.3 & 77.0 & 92.4 & 100.0 \\
1994 & 28.6 & 57.2 & 57.2 & 57.2 & 57.2 & 71.5 & 85.8 & 92.9 & 100.0 \\
1996 & 9.1 & 45.5 & 54.6 & 63.7 & 63.7 & 63.7 & 72.8 & 91.0 & 100.0 \\
\hline
\end{tabular}

Fuente: Consejo Nacional Electoral del Ecuador (CNE), Asamblea Nacional del Ecuador.

De esta información mostrada, se desprende el enorme peso que tenían los bloques legislativos pequeños, cuyos votos pasan a ser vitales dentro del juego político parlamentario, lo que, a su vez como una forma de retroalimentación, se convierte en un incentivo para la proliferación de partidos. Si a esto se añade el efecto de algunas disposiciones electorales, como la utilización de la fórmula proporcional para la asignación de escaños legislativos $y$, posteriormente, la utilización del sistema de votación personalizado en listas abiertas, estas serían entre otras, las causas de la fragmentación. El resultado inevitable de esta fragmentación del sistema de partidos ha imposibilitado de que un partido (Gráfico № 3), ya sea de gobierno o de oposición, pueda alcanzar la mayoría tanto en el Legislativo como en la primera vuelta presidencial ${ }^{9}$.

En el análisis de las causas que han configurado esta situación se destaca la utilización de la fórmula proporcional en las elecciones legislativas; como lo han demostrado varios estudios comparativos (Nohlen, 1995) o (Taagepera y Shugart, 1989), tiende a desembocar en una representación fragmentada e impide la conformación de mayorías claras. Dentro del debate, subyace la teoría de que esta imposibilidad de conformar mayorías, se atribuye a la realización de las elecciones legislativas concurrentemente con las elecciones presidenciales, esto es en primera vuelta ${ }^{10}$.

Gráfico $\mathbf{N}^{\mathbf{0}} 3$ Fuente: CNE. Porcentaje de partidos políticos en legislativo vs. ejecutivo

\footnotetext{
9 Como se puede ver en el cuadro número 3, a lo largo de todo el período ningún partido ha conseguido más de la mitad de los escaños legislativos. Tanto en las elecciones generales, que se realizan conjuntamente a la primera vuelta presidencial, como en las de medio período, que tuvieron vigencia hasta 1998, la primera bancada apenas ha ocupado en promedio la tercera parte de los escaños, con un tamaño máximo de $44.9 \%$ y un mínimo de $22.2 \% 6$.

${ }^{10}$ Esta es solamente una verdad parcial, ya que si bien es cierto que en la única ocasión en que se realizaron elecciones legislativas en la segunda vuelta presidencial (1979) se conformó el bloque legislativo que ha estado más cerca de lograr la mayoría, también se debe considerar que al mismo tiempo hubo 10 listas con representación y que las bancadas de menos de tres diputados equivalían al $60.0 \%$ del total del Legislativo. Por otra parte, en las elecciones de medio período no ha habido un comportamiento diferente. Se trata, por tanto, de una causa necesaria pero no suficiente, a la que deben añadirse las mencionadas antes.
} 
$*=$ elecciones legislativas de medio periodo.

\begin{tabular}{cccccc}
\hline $\begin{array}{c}\text { Año de } \\
\text { elecciones }\end{array}$ & $\begin{array}{c}\text { \% de escaños } \\
\text { legislativo }\end{array}$ & $\begin{array}{c}\text { Partido político o } \\
\text { bancada legislativa }\end{array}$ & Tendencia & Presidente & $\begin{array}{c}\text { Partido político } \\
\text { presidente }\end{array}$ \\
\hline $\mathbf{1 9 7 9}$ a 1981 & 44.9 & CFP & Gobierno & Roldos & CFP \\
$\mathbf{1 9 8 1}$ a 1984 & 33.8 & ID & Oposición & León Febres & PSC \\
$\mathbf{1 9 8 4}$ & 23.9 & ID & Oposición & & \\
$\mathbf{1 9 8 6}^{*}$ & 42.3 & ID & Gobierno & Rodrigo Borja & ID \\
$\mathbf{1 9 8 8}$ & 22.2 & PSC & Oposición & & \\
$\mathbf{1 9 9 0 *}$ & 27.3 & PSC & Oposición & Sixto Durán & PUR \\
$\mathbf{1 9 9 2}^{\mathbf{1 9 9 4} *}$ & 32.5 & PSC & Oposición & & \\
$\mathbf{1 9 9 6}$ & 32.9 & PSC & Oposición & Abdalá Bucaram & PRE \\
\hline
\end{tabular}

Elaborado por: Grupo de Investigación.

Es esta fragmentación del Legislativo, así como, la ausencia de regularidad en las elecciones presidenciales son el reflejo de un conjunto de factores, tanto institucionales como estructurales, que determinan las pautas de la votación y de las normas que rigen los procesos electorales y al propio sistema de partidos ecuatoriano (Mejía, 2002) (Freidenberg, 2000) (Mainwaring y Scully, 1995) (Conaghan, 1994).

Todo esto como insumos para obtener, finalmente, altos niveles de volatilidad ${ }^{11}$, colocando al Ecuador según Mainwaring y Scully (1995) con uno de los índices más altos en América Latina $^{12}$

En tercer lugar, la polarización ideológica es otro de los signos que se destacan dentro del sistema de partidos ecuatoriano (Freindenberg y Alcantara, 2001).

\footnotetext{
11 Si se considera que la volatilidad mide los cambios absolutos de la proporción de votación; o escaños obtenidos de cada partido o de alianzas, se puede sostener que se trata fundamentalmente de una expresión de la conducta de los electores. Pero, al mismo tiempo, dado que existe correlación entre la fuerza de cada partido y el arraigo en la sociedad, es también una expresión de su debilidad y una manifestación adicional de la escasa institucionalización del sistema de partidos.

12 En la medición de Mainwaring y Scully (1995: 8), el Ecuador ocupa el tercer lugar en volatilidad media, después de Brasil y Perú. Los niveles más altos se encuentran en las elecciones presidenciales, mientras que en las legislativas tiende a moderarse; lo que podría interpretarse como una manifestación de cierta fuerza y arraigo de los partidos, así como de la coexistencia de lógicas particulares de cada tipo de elección. Las elecciones presidenciales son caracterizadas por una mayor personalización.
} 
Tanto la auto ubicación que hacen los miembros de cada uno de los partidos, como la asignación de posiciones hacia los otros partidos, coinciden en establecer distancias relativamente amplias entre ellos.

Así, en el año 1996, dentro de una escala de izquierda a derecha (1 a 10), la ubicación que se asignaban a sí mismos los políticos de cada partido ocupaba un rango que iba desde 2 , en la izquierda, hasta 7.1, en la derecha. Mientras, a sus respectivos partidos, les asignaban valores aún más extremos, en un rango que iba desde 1.7 hasta 7.2 (Ibídem).

Gráfico $\mathbf{N}^{\mathbf{0}} 4$ Votación por tendencias en 1ra. Vuelta Fuente: CNE, Freidenberg (2001).

\begin{tabular}{ccccc}
\hline $\begin{array}{c}\text { Año/ } \\
\text { Tendencia } \\
\text { Ideológica (\%) }\end{array}$ & $\begin{array}{c}\text { Izquierda- } \\
\text { Centroizquierda }\end{array}$ & $\begin{array}{c}\text { Derecha- } \\
\text { centroderecha }\end{array}$ & Populismo ${ }^{\mathbf{3}}$ & Otros \\
\hline $\mathbf{1 9 7 9}$ & 16.7 & 46.6 & 27.7 & 9.0 \\
$\mathbf{1 9 8 4}$ & 52.5 & 27.2 & 13.5 & 6.8 \\
$\mathbf{1 9 8 8}$ & 41.2 & 16.3 & 25.5 & 17.0 \\
$\mathbf{1 9 9 2}$ & 15.4 & 57.8 & 23.6 & 3.2 \\
$\mathbf{1 9 9 6}$ & 23.0 & 40.7 & 26.3 & 10.0 \\
\hline
\end{tabular}

Elaborado por: Grupo de Investigación.

Por su parte, la percepción de la ubicación de los otros partidos, mantenía la distancia entre los puntos extremos, aunque se desplazaba hacia la derecha ya que se movía entre 3 y 9.4 . En términos generales, y con la excepción de un partido: Democracia Popular (DP), estas percepciones no habían sufrido alteraciones significativas a lo largo del tiempo en las mediciones. Sin duda, que esta información al tratarse de percepciones ideológicas ${ }^{14}$ de los actores políticos, constituyen pautas y referencias para la acción, siendo también, un

13 Respecto al "populismo", René Mayorga (1995) plantea establecer un concepto estructurado alrededor de un núcleo significativo de tres dimensiones: 1) una dimensión política en cuanto a movimiento social conformado alrededor de líderes carismáticos que no se apoyan en estructuras intermedias partidarias, sino precisamente en la movilización de sectores populares; 2) una dimensión ideológica definida por una orientación nacionalista de identificación del Estado con el pueblo; $y, 3)$ un eje de política económica caracterizado por la puesta en práctica de política, tanto de control estatal de sectores económicos considerados estratégicos como de redistribución de ingresos. Véase el estudio en Mayorga, R.(1995), Antipolítica y neopopulismo, CEBEM, La Paz.

14 Giovanni Sartori $(1987 ; 1980)$ incorpora a su análisis la dimensión ideológica y concluye que mientras la pequeña distancia refuerza la base consensual de la democracia, los partidos que compiten hacia los extremos del espectro político la erosionan. De este modo, existen sistemas compatibles con la democracia (sistemas predominante, bipartidista, pluralista moderado, pluralista polarizado y atomizado) y sistemas menos competitivos (sistemas unipartidista y hegemónico) (Sani y Sartori, 1983). 
componente importante para determinar una mayor o menor predisposición para establecer alianzas o, incluso, cualquier tipo de acercamiento y acuerdo con los otros partidos, dependiendo en buena medida, de la percepción acerca del lugar que cada uno ocupa en el espacio ideológico. En esta situación de polarización ${ }^{15}$ multipartidista (Sani y Sartori, 1983) como la que ponen en evidencia las brechas observadas, existen pocas posibilidades para la construcción de la política pública conjunta y para evitar una separación de poderes Ejecutivo-Legislativo, impulsando prácticas confrontacionales y movimientos centrífugos (Malamud, 2003;Duverger,1957).

Asimismo, los lugares extremos no están ocupados por partidos marginales, sino por algunos de los que han mantenido altos niveles de votación a lo largo del periodo entre 1979 y 1986, quienes consecuentemente contaron con una importante representación parlamentaria, así como, por los que han jugado un papel central en la conformación del gobierno o de oposición (Ibídem). Por tanto, la polarización se ha derivado en las posiciones de los partidos que dentro de la tipología de Sartori (1992) cuentan para la conformación de gobiernos o de la oposición, esto es, de los partidos políticos que a lo largo del tiempo tienen posibilidades de coalición o de gobierno, por el contrario, de chantaje. En definitiva, esta propensión estructural al conflicto entre el Ejecutivo con el Legislativo, en un sistema multipartidista débil ${ }^{16}$ con raíces históricas que muestra la realidad de que ningún partido obtiene más allá del $40 \%$ de los votos en las elecciones legislativas (en el periodo entre 1979 al 1996), parece ser que coexisten tantos votantes indecisos, como políticos indecisos (Conaghan, 1995). No obstante, es importante tener en cuenta como el sistema político, el sistema de partidos y las reglas electorales confluyen para que los actores políticos se autodefinan como anti-partidos (Ibídem).

\section{La destitución de Bucaram: Caso de estudio.}

El 10 de agosto de 1996, Abdalá Bucaram Ortiz, en investido como Presidente del Ecuador. Desde aquel momento, varios son los escenarios adversos que tiene que enfrentar sumándose a una crisis política ocasionada por la agenda de políticas públicas planteada y a las medidas económicas que se implementaron. Luego de movilizaciones organizadas por organizaciones civiles y partidos de oposición, el Congreso Nacional convoca a una Sesión extraordinaria para analizar la crisis política que vivía Ecuador. En esta sesión del 6 de

15 Según Sani y Sartori (1983) la polarización es una de las dimensiones más relevantes del sistema de partidos, ya que constituye la variable con mayor valor explicativo a la hora de representar el comportamiento del sistema democrático, su eficacia, actividad y viabilidad. Este mecanismo da cuenta de la distancia que separa en el espectro ideológico a los partidos de un sistema dado, midiéndose, habitualmente por la distancia que separa a los partidos en atención a la dimensión ideológica izquierda-derecha.

${ }^{16}$ Conaghan (1993) toma esta definición de McDonald (1971:32-92). Como ejemplo, cabría que señalar que los socialdemócratas (Izquierda Democrática) estuvieron a punto de superar el umbral del $40 \%$ en las elecciones legislativas de 1988, obteniendo veintinueve (29) de los setenta y un escaños (61). Sin embargo, la ID obtuvo unos pobres resultados en las elecciones a medio mandato de 1990 y en las legislativas de 1992. 
febrero de 1997, el Congreso Nacional, remueve al Presidente por "incapacidad mental para gobernar"17, nombrándose, por mayoría ${ }^{18}$ simple de votos al entonces Presidente del Congreso Nacional: Fabián Alarcón, como Presidente Interino del Ecuador; inexistente jurídicamente en la Constitución vigente de ese espacio temporal ${ }^{19}$.

Es así que, el diputado Franklin Verduga, legislador opositor y miembro del Partido Social Cristiano $^{20}$, presentó un proyecto de resolución en la sesión del 6 de febrero de 1997. Su moción perseguía, como objetivos: a) declarar cesante al Presidente de la República y, además, b) reemplazarlo por el Presidente del Congreso, c) llamar a elecciones anticipadas para todas las dignidades de elección popular, incluyéndose en la propuesta el d) concluir las funciones de autoridades públicas como el Contralor, Fiscal, Procurador, Superintendentes y miembros del Tribunal de Garantías Constitucionales y, e) allanar el camino legal y político para la convocatoria a una Asamblea Constitucional para la reestructuración del Estado ${ }^{21}$. En este sentido, es evidente que "el Congreso Nacional atentó contra la continuidad del sistema democrático consagrado en la normativa constitucional y legal, cesando al Presidente Bucaram por motivos en los cuales debía mediar un juicio político; no se respetó el debido proceso y no se motivó debidamente la declaración de

${ }^{17}$ El Acta N ${ }^{\circ} 1$ de la Sesión Extraordinaria del 6 de febrero de 1997 se puede descubrir lo ocurrido en aquel día en el Congreso Nacional. Así, con 44 (cuarenta y cuatro) votos se destituyó al Presidente Bucaram. Existiendo 34 votos en contra que corresponden a los diputados afines al partido de gobierno. La oposición, basados en lo establecido en el literal d) del artículo 100 de la Constitución de 1979 codificada en 1996, destituyó al presidente Bucaram. El texto de la Constitución Política de la República del Ecuador de 1979, Codificada en 1996, publicada en el Registro Oficial N 969, del 18 de junio de 1996 dice textualmente: "Art. 100.- El Presidente de la República cesará en sus funciones y dejará vacante el cargo: a. Por terminación del período para el cual fue elegido; b. Por muerte; c. Por renuncia aceptada por el Congreso Nacional; d. Por incapacidad física o mental declarada por el Congreso Nacional; y, e. Por destitución o abandono del cargo declarado por el Congreso Nacional".

${ }^{18}$ El Reglamento Interno de la Función Legislativa de 1996, en su artículo 74, determinaba que: "Para la aprobación de todo asunto en el Congreso Nacional se requiere de la mayoría absoluta de los legisladores votantes, a menos que [en] la Constitución o este Reglamento se establezca otra mayoría (...)". Es decir, que permitir una mayoría simple para aceptar la moción de destitución del Presidente fue improcedente desde el punto de vista Constitucional y legal.

19 La Constitución de 1979 vigente en ese momento no señalaba el ¿cómo debía realizarse la sucesión presidencial en caso de ausencia definitiva del Presidente?, ya que solo establecía su procedimiento en caso de ausencia temporal. Los vacíos legales permitieron que la sucesión sea inconstitucional; no quedaba claro quién debía subrogar y asumir definitivamente la Presidencia. Finalmente, la disputa centró entre la Vicepresidenta Rosalía Arteaga y el Presidente del Congreso Fabián Alarcón, siendo éste último quien fue nombrado como Presidente interino del Ecuador. El Legislativo, es decir, la mayoría simple de legisladores, se ampararon en el segundo inciso del Art. 1 de la Codificación Constitucional para proclamar a Fabián Alarcón como presidente interino. Este artículo decía: "La soberanía radica en el pueblo que la ejerce por los órganos del poder público". El Legislativo asumió que los legisladores eran los soberanos mandantes y representantes del pueblo.

20 Uno de los Partidos políticos más importantes en Ecuador desde el retorno a la democracia. Para su caracterización e influencia véase Freidenberg, F. y Alcantara M., (2001), Los dueños del poder. Los partidos políticos en Ecuador (1978-2000), FLACSO, Quito.

${ }^{21}$ Véase moción de censura en el Acta $N^{\circ} 1$ de la Sesión Extraordinaria Vespertina del Congreso Nacional, del 6 de febrero de 1997 (Asamblea Nacional del Ecuador, 2017). 
incapacidad mental, volviendo nulo e inconstitucional lo actuado por el Legislativo. En definitiva, la Función Legislativa dio un golpe de Estado, al destituir al Presidente de la República y revocar el mandato de autoridades elegidas por la ciudadanía en 1996" (Banegas, 2014:24). Este ejemplo sirvió como motivación, si cabe el término, para crear normas más específicas sobre la cesación del cargo presidencial y evitar vacíos legales. Como resultado, en 1998 se redactó una nueva Constitución que buscó reforzar el poder del presidente (Salgado, 2012), sin embargo, se afianzó el problema de doble legitimidad (Linz,1993), ahondando la confrontación entre el Ejecutivo con el Legislativo y minando los incentivos para cooperar con él (Mejía Acosta y Polga-Hecimovich, 2011). En este escenario de mutua desconfianza y atrapados en una estructura del dilema del prisionero, en donde no podrán cooperar, aunque lo deseen hasta encontrar una forma de neutralizar la mutua desconfianza y cuando se establezca la necesidad de cooperación (Axelrod, 1986). Si bien es cierto esta confluencia hacia la cooperación estratégica entre Ejecutivo y Legislativo no implica ausencia de conflicto, sin embargo, es posible considerar que si se requiere la existencia de cierto margen de interés común. Pero del ejemplo anterior, en la decisión de la mayoría simple de legisladores que, de los resultados posibles del juego, de la interacción y de la votación final por la destitución de Bucaram, no existió la posibilidad de un pago eficiente para todos sino sólo para uno de los contendientes (Ibídem). Así, el bloque de Legisladores de oposición en un juego de conflicto total, sumaron sus intereses en contraposición de los adversarios (Ejecutivo), encontrándose en un escenario de oposición frontal (Brams, 1994). En ese contexto, el bloque opositor de los legisladores del PSC (32\%), contaba con el apoyo del legislador de veto y del legislador mediano (42\%), produciéndose a la postre un "gobierno de la legislatura" (Colomer \& Negretto, 2005:79), mientras el partido de Bucaram se mantenía en una situación de minoría (26\%).

Gráfico No 5 Fuente: Colomer \& Negretto (2005).

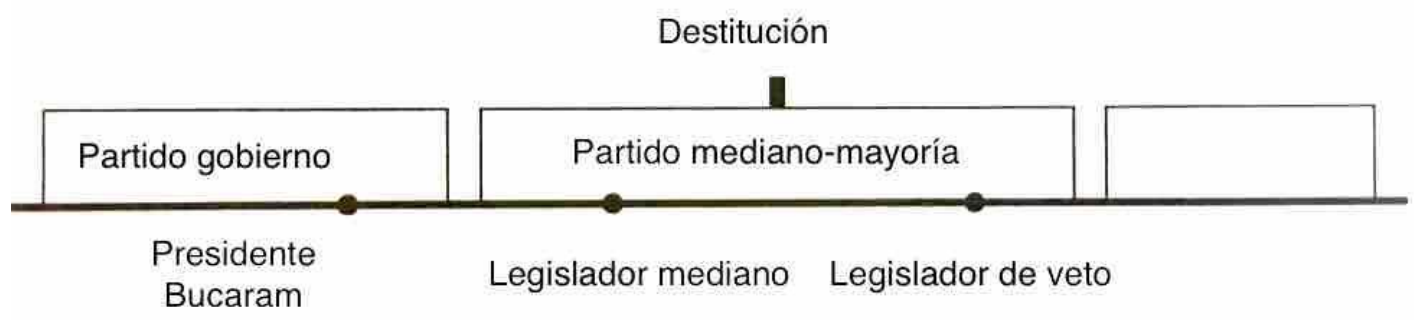

Elaborado por: Grupo de Investigación.

Siguiendo esta línea y, encontrándonos en una situación de polarización del manejo de la agenda legislativa frente al Ejecutivo; más aún si la regla de mayoría simple estableció que la proposición de destitución de Bucaram sea resuelta por este mecanismo, contando con el voto favorable de la mitad más uno de los integrantes de los legisladores asistentes a la 
sesión del 6 de febrero de 1997, en términos más formales, del primer número entero superior a N/2. La mayoría simple de legisladores medianos (otros partidos) y de veto (PSC), así definida para el propósito de este trabajo, aseguró que si el grupo de oposición a Bucaram aprobaría la propuesta de destitución no sería aprobada la alternativa contraria ${ }^{22}$. Formalmente, si se aprueba "A" no se aprobará "no A". En la práctica y previamente a este escenario, el PSC o legislador de veto se enfrentó a dos alternativas: a) apoyar políticamente al nuevo gobierno, aun cuando ello implicaría avalar el reciente triunfo de Bucaram sobre $\mathrm{Nebot}^{23}$; quien fuese candidato a Presidente por este partido en el reciente proceso electoral, o b) mantenerse en una posición neutral y a cierta distancia; de cualquier forma la estrategia de la oposición legislativa (PSC fundamentalmente) era esperar a que Bucaram expusiera una política pública importante que impulse a los partidos que ostentaban la representación del votante mediano a sumarse a su partido de oposición (o partido de veto) a posiciones que asumieran la necesidad irremediable de reconocer, en mayor medida, la consecuente disputa en la agenda legislativa y de la instauración del gobierno legislativo (Ibídem), o en este caso de estudio, la destitución del Ejecutivo. (Ver gráfico 6).

Gráfico No 6- Tabla de preferencias de Legislativo y Ejecutivo. Destitución de Bucaram.

Preferencias Legislador Veto (PSC)

\section{Preferencias Ejecutivo}

\begin{tabular}{llll}
\hline PSC & Bucaram & PSC & Bucaram \\
\hline 4 Neutralidad & No destitución & 4 Apoyo & No destitución \\
3 Neutralidad & Destitución & 3 Neutralidad & Destitución \\
2 Apoyo & Destitución & 2 Neutralidad & No Destitución \\
1 Apoyo & No destitución & 1 Apoyo & Destitución \\
\hline
\end{tabular}

Elaborado por: Grupo de Investigación.

Este juego del Legislativo y Ejecutivo, tanto en la destitución de Bucaram como en la configuración de la política pública, tiene la estructura del Dilema del prisionero (ver el

\footnotetext{
22 Esta propiedad, aplicada a las situaciones en donde hay dos alternativas, es decir situaciones binarias, asegura que la regla llega a una decisión y que la misma tendrá consistencia lógica. La mayoría simple es el menor número necesario para lograr la decisividad y la logicidad del resultado.

${ }^{23}$ En la segunda vuelta electoral, Bucaram consiguió el apoyo de diferentes sectores entre los que destacaban los movimientos sociales y, en particular, los grupos indígenas representados en la CONAIE y en MUPP-NP. Asimismo, los candidatos también buscaron el voto de los sectores laborales y de los informales. Finalmente, el triunfo del líder populista sorprendió tanto a la clase política ecuatoriana como a la ciudadanía, que esperaban que los socialcristianos se hicieran con la Presidencia de la República (Freinderber, 2001).
} 
Gráfico $\mathrm{N}^{\mathrm{o}} 7$ ). Ambos actores tienen una estrategia dominante ${ }^{24}$, la cual produce un primer resultado, el de la casilla inferior izquierda $(4,3)$. El PSC siempre tiene como estrategia dominante la neutralidad, pues esta le da un mejor resultado en su posición de partido mayoritario y de partido de veto en el Legislativo. Igualmente, Bucaram tiene su propia estrategia dominante, que es la de no ser destituido, a pesar de no contar con el votante mediano, ni mayoría en el Legislativo, la neutralidad del partido de veto (PSC) a la propuesta le permitía, a largo plazo, cambiar esta configuración. Con el resultado que se produce a partir de las estrategias dominantes, ambos jugadores tienen éxito en el sentido de que toman la decisión que quieren en primer lugar (neutralidad-destitución) entre sus dos alternativas. Esto sólo era posible mediante la cooperación de los dos adversarios, sin embargo, al cambiar de jugada el PSC, el resultado del juego se traslada hacia la casilla superior izquierda, como lo indica la flecha (Gráfico $\mathrm{N}^{\mathrm{o}} 7$ ), en donde evidentemente el nivel de eficiencia es más bajo para ambos: Legislativo; PSC fundamentalmente, y Ejecutivo en la relación de preferencias $(2,1)$ para cada uno.

Gráfico No 7. Caso de estudio (Bucaram): Legislativo versus Ejecutivo y resolución de destitución.

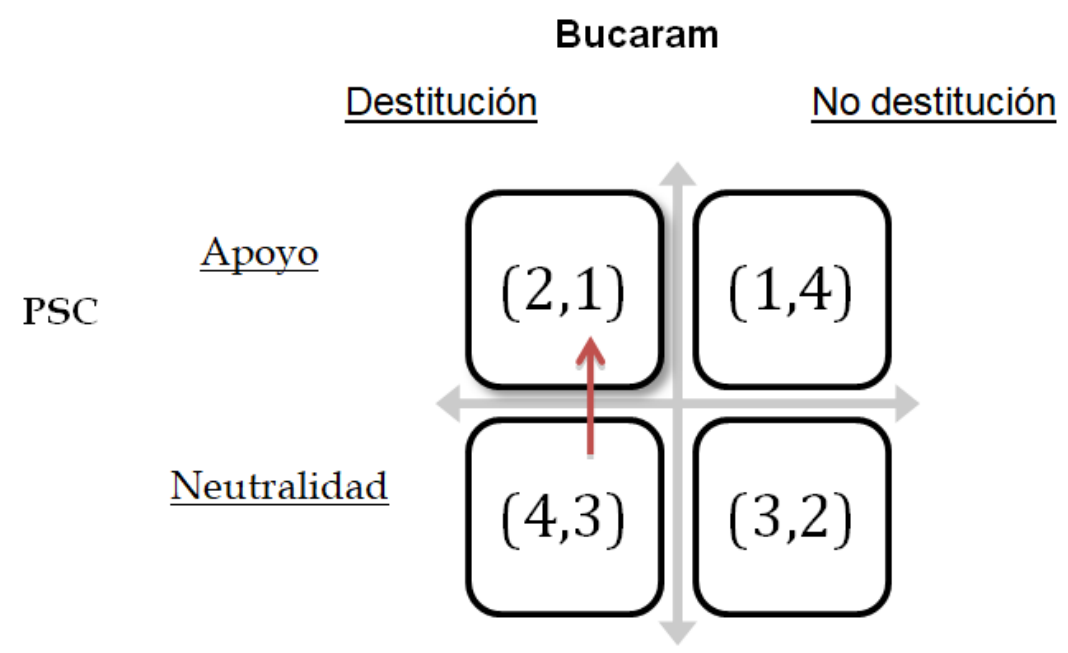

Elaborado por: Grupo de Investigación.

\section{Conclusiones.}

- Como se ha señalado en el desarrollo de este trabajo académico, el sistema de partidos ecuatoriano está caracterizado por graves problemas de articulación a nivel de las masas y de las élites, en el cual, no hay votantes indecisos sino políticos indecisos (Freindenberg et. al, 2001). En ese contexto, los problemas de articulación

\footnotetext{
24 Por definición, una estrategia es dominante cuando constituye la mejor respuesta de un jugador ante cualquiera de las estrategias posibles de su oponente. Frente a una estrategia dominante, las otras estrategias entre las que puede elegir son dominadas, ya que implican resultados claramente inferiores a la primera.
} 
no se limitan solamente a los nacientes partidos populistas ecuatorianos o a los antiguos partidos, sino que también han afectado a los modernos partidos marxistas, socialdemócratas y democristianos, aunque quizás en menor grado; dados los resultados electorales. Además, el carácter sumamente instrumental de los vínculos de las élites con los partidos, fomenta un comportamiento impredecible (Conaghan, 1994) y acrecienta la volatilidad en las relaciones Ejecutivo y Legislativo.

- Igualmente, la debilidad del sistema ecuatoriano de partidos tiene profundas raíces históricas; su completo análisis no es el alcance de este trabajo. Sin embargo, es importante tener en cuenta como las reglas electorales; una característica de antipartidos entre los actores políticos, han afectado al desarrollo de los partidos, reforzando así el síndrome de tensión institucional (Conaghan, 1994). Así, esta propensión estructural al conflicto Ejecutivo y Legislativo se agrava también, en la renaciente democracia ecuatoriana, debido a la existencia de un sistema multipartidista débil, esto es, un sistema de partidos muy denso en el que ningún partido fue capaz de hacerse con más del $40 \%$ de los escaños parlamentarios en las elecciones legislativas en el periodo propuesto (1979 a 1996).

- En este periodo abordado y con la alta fragmentación política mostrada, la formulación de políticas públicas ha transcurrido en una permanente alternancia entre la rigidez y la volatilidad de los acuerdos entre el Ejecutivo y el Legislativo. En el primer caso, la falta de adaptabilidad del sistema proviene de la incapacidad de los actores para encontrar puntos mutuos de acuerdo y establecer alianzas. En el segundo escenario, la volatilidad ha resultado de los esfuerzos esporádicos para romper con la rigidez e impulsar agendas o reformas a través de acuerdos selectivos. Es justamente este péndulo, que oscila de la rigidez a la volatilidad en las relaciones Ejecutivo - Legislativo y en la construcción de las políticas públicas, el que explica buena parte del proceso político en el periodo abordado.

- Además, la tradicional conformación de las alianzas fantasma entre el Ejecutivo y el Legislativo o con los partidos políticos, durante la etapa ilustrada en este trabajo (1979-1996), resume precisamente la necesidad de los actores de formar acuerdos clandestinos y circunstanciales en lugar de hacer acuerdos transparentes y de largo plazo ayudados por la ausencia de mecanismos imparciales de arbitraje, como una Corte Suprema de Justicia o Tribunal Constitucional. Así, la legalidad y la ejecución de los acuerdos políticos han estado sujetas a los vaivenes electorales y a la discrecionalidad de sus actores. Muchas veces inconstitucionalmente o sin el debido proceso, como en el caso abordado del ex presidente Bucaram.

- Hemos caracterizado al presidencialismo ecuatoriano, en donde, el Ejecutivo es fuerte pero muy limitado, sujeto a muchas contingencias en el ejercicio de su poder, mientras que un Legislativo relativamente débil dispone de amplias capacidades y múltiples disposiciones de maniobra para ejercer su poder e influir en el gobierno. En tal sentido, cabría identificar de tribuno al Legislativo, en la medida que puede hacer mucho; como destituir Presidentes, pero puede impedir poco; dadas las atribuciones establecidas en la Ley. Pero al mismo tiempo, esta correlación entre el Ejecutivo y la capacidad del Legislativo para formular e implementar la política pública operan inversamente, de tal manera que, la falta o debilitamiento en el control o ejercicio del gobierno por parte del Ejecutivo repercute en un aumento de las fuerzas del Legislativo. De hecho, nada acrecienta más, a la articulación del 
legislador de veto con el legislador mediano, que la falta de capacidad o ejercicio de gobierno por parte del Ejecutivo.

- Esto nos obliga a reconocer que, en la construcción de la política pública, no es tanto la conflictividad de los actores políticos la que se encuentra investida, cuanto la conflictividad inherente al Ejecutivo con el Legislativo que los lleva a mantener lo que consideramos su estructural enfrentamiento. Y por eso también, el análisis de la conflictiva agenda política, no puede dejar de centrarse en el carácter institucional del conflicto político, aun cuando no se excluya su protagonización a los mismos actores políticos (PSC en el caso de este trabajo).

- Finalmente, la inestabilidad creada en el efímero gobierno de Abdalá Bucaram (agosto de 1996-febrero de 1997), así como su destitución por parte del Legislativo, ayudaron a colocar a otro instrumento de disputa de la agenda pública en el centro del debate político: la Asamblea Constituyente. Como hemos mostrado en el caso de estudio: a) la forma en que se procedió para esta destitución, b) la interpretación del texto constitucional; facilitada en buena medida por sus vacíos, c) la designación de un Presidente interino sin apego a las disposiciones constitucionales y legales, d) la alteración del sistema electoral y e) la necesidad de encontrar legitimidad social y política para los actores políticos fueron los factores de mayor peso para que la idea de la Asamblea Constituyente se cristalice. En la práctica, se impuso la percepción de que el sistema político se había roto o, que por lo menos, se necesitaba mejorar el grado de institucionalización de los partidos políticos y la polarización ideológica del sistema y otros vinculados a la capacidad de aprendizaje de las dirigencias partidarias y su vocación por negociar agendas de políticas públicas o de gobierno para evitar que se produjeran nuevamente las disputas Ejecutivo-Legislativo con los desenlaces mostrados como el de Bucaram. Luego de un referéndum en 1997 y la implementación de la Asamblea Constituyente en 1998, Jamil Mahuad, el siguiente presidente electo democráticamente en las urnas, también era depuesto.

\section{Referencias bibliográficas.}

Axelrod, R., (1986), La evolución de la cooperación, Alianza Editorial, Madrid.

Banegas, M., (2014), La Muerte Cruzada en el Nuevo Texto Constitucional, Relaciones Ejecutivo-Legislativo, Creative Commons, UASB. Digital

Brams, S., (1994), Theory of moves. Cambridge University Press.

Balda, R. (2008), "Hacia un nuevo sistema de gobierno: diseño institucional del presidencialismo en la nueva Constitución del Estado ecuatoriano”, en Ávila et al., Desafios constitucionales: La Constitución ecuatoriana del 2008 en perspectiva, Ministerio de Justicia y Derechos Humanos, Quito. 
Bustamante, F., (1997), "La cultura política y ciudadana en el Ecuador”, en varios Autores, Ecuador: un problema de gobernabilidad, CORDES, Quito.

Conaghan, C., (1994) "Loose Parties, Floating Politicians and Institutional Stress: Presidentialism in Ecuador; 1979-1988”, en Linz, J. y Valenzuela A., (editores), The Failure of Presidential Democracy, Johns Hopkins University Press.

Conaghan, C., (1995), "Politician Against Parties: Discord and Disconnection in Ecuador's Party System", en Maiwaring, S. \& Scully, T., Building Democratic Institutions, Stanford University Press, Stanford.

Duverger, M, (1957), Los partidos políticos, Fondo de cultura económica, México.

Duverger, M. (1970), Instituciones Políticas y Derecho Constitucional, Editorial Uninorte, Madrid.

Freidenberg, F. y Alcántara M., (2001), Los dueños del poder. Los partidos políticos en Ecuador (1978-2000), Flacso, Quito.

Lijphart, A., (1999), Patterns of Democracy. Yale University Press, New Haven and London.

Linz, J. (1993), “Los peligros del presidencialismo”, en Formas de Gobierno: Relaciones Ejecutivo - Parlamento, Comisión Andina de Juristas, Lima.

Linz J., y Valenzuela A., (1993), Las crisis del presidencialismo 1. Perspectivas comparadas (Vol. 1), Alianza Universidad, Madrid.

Linz J. y Valenzuela A. (1994), La crisis del presidencialismo. 2. El caso de Latinoamérica, Alianza Editorial, Madrid.

Mainwaring S. \& Scully T., (1995), Building Democratic Institutions. Party Systems in Latin America, Stanford University Press.

Malamud, A., (2003), "Partidos políticos”, en Introducción a la Ciencia Política 4ta edición, Pinto J., (compilador), Eudeba, Buenos Aires. 
Mejía, A., (2002), Gobernabilidad democrática, KAS, Quito.

Mejía, A. y Polga-Hecimovich, J., (2011), "Coalition Erosion and Presidential Instability in Ecuador" en Latin American Politics and Society, Vol. 53 № 2, (p: 87-111).

Nohlen, D. (1991), "Sistemas de Gobierno: perspectivas conceptuales y comparativas”, en Dieter Nohlen et. al, Presidencialismo versus Parlamentarismo en América Latina, Editorial Nueva Sociedad, Caracas.

Nohlen, D., (1993), Sistemas electorales de América Latina, Fundación Friedrich Ebert, Lima.

Pachano, S., (2003a), El tejido de Penélope, en Reforma Política en América Latina. KAS. Brasil.

Payne, J. (2006), "El equilibrio de poder entre el Ejecutivo y el Legislativo: papel de la Constitución y los partidos políticos" en La Política importa, Democracia y desarrollo en América Latina. Editorial Planeta, Washington.

Pérez Liñán, A., (2009), Juicio político al presidente y nueva inestabilidad política en América Latina, Fondo de Cultura Económica, Buenos Aires.

Riker, W., (2001), “Teoría de juegos y de las coaliciones políticas”, en Diez textos básicos de Ciencia política, Editorial Ariel, España.

Sánchez, F., (2008), ¿Democracia no lograda o democracia malograda? Un análisis del sistema político del Ecuador: 1979-2002, FLACSO, Quito.

Sani, G., \& Sartori, G. (1983), "Polarization, fragmentation and competition in Western democracies", en Daader y Mair (eds.), Western European party systems. Continuity and change, Londres, (307-340)

Sartori, G., (1987), ¿Qué es la democracia?, Taurus, Buenos Aires.

Sartori, G. (1992), Partidos y sistemas de partidos, Editorial Alianza 2a . Edición, Madrid. 
Sartori, G. (2003), Ingeniería Constitucional Comparada; Una investigación de estructuras, incentivos y resultados, con el posfacio: La transición de México, ¿hacia dónde? Una agenda para la Reforma, Fondo de Cultura Económica, México.

Taagepera, R. \& Shugart, M., (1989), Seats \& Votes. The Effects \& Determinants of Electoral Systems, Yale University Press, Binghamton.

Valles, J. y Martí Puig, S., (2015), Política un manual, Nueva edición actualizada, Huertas Industrias Gráficas, España. 
Para citar el artículo indexado.

Dahik I., Del Pozo R., Galarza J. \& Rivadeneira E. (2018). Ejecutivo y legislativo: la inacabada disputa del poder en el sistema político ecuatoriano. Revista electrónica Ciencia Digital 2(2), 181-199. Recuperado desde: http://cienciadigital.org/revistacienciadigital2/index.php/CienciaDigital/article/view/82/76

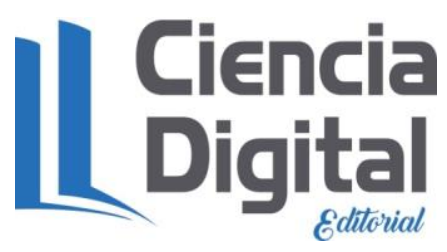

El artículo que se publica es de exclusiva responsabilidad de los autores y no necesariamente reflejan el pensamiento de la Revista Ciencia Digital.

El articulo queda en propiedad de la revista y, por tanto, su publicación parcial y/o total en otro medio tiene que ser autorizado por el director de la Revista Ciencia Digital.
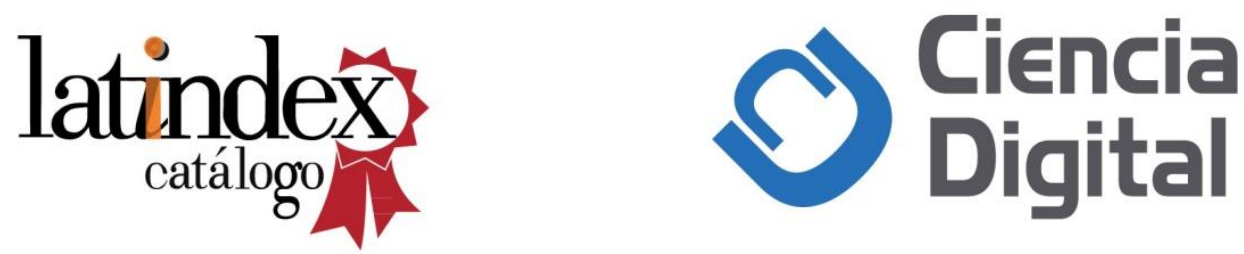